ziehung zu dem vorliegenden Thema steht eine Mitteilung von FrIEDRICH aus der Bergmannschen Klinik, der eine Tonusabnahme des Magens bei Entleerung kleinster Flüssigkeitsmengen aus der Blase nachweisen konnte, nachdem schon BERgmann darauf hingewiesen hatte, daß Magenschmerzen nach dem Urinieren nachlassen können. Wenn man nun berücksichtigt, da $\beta$ Veränderungen in der Prostata sehr häufig zu einer Hypertonie der Blase führen, so rückt die Annahme durchaus in das Bereich des Vorstelibaren, daß die Tonusrelation von Blase und Kolon das Substrat des lilinisch beobachteten Einflusses einer Prostatitis auf die Darmmotilität darstellt.

\section{ZUR ENTSTEHUNG UND BEHANDLUNG DER MASTDARMFISTELN}

\section{Von}

Oberarzt Dr. L. RickmanN.

Aus dem Sanatorium für Lungenkranke in St. Blasien. (Leitender Arzt: Prof. Dr. BACMEISTER.)

Uber die Entstehung der Mastdarmfisteln, insbesondere über ihre Beziehungen zur Tuberkulose ist schon seit alter Zeit gestritten worden. Durch harte Kotpartikel, die sich durch Stagnation, Eindickung und Verhärtung des Darminhaltes im unteren Teile des Mastdarmes bilden, kommt es zu Excoriationen, durch welche in den Fäces vorhandene Mikroorganismen verschiedenster Art eindringen und bei der außerordentlich großen Resorptionsfähigkeit und dem regen Stoffwechsel dieser Partie in die Wand geführt werden. LACHMAN' und SMITH erbrachten als erste den Nachweis von Tuberkelbacillen in Analfisteln und bewiesen dadurch den Zusammenhang zwischen Analfistel und Tuberkulose. IAANz glaubt trotzdem, daß die Mastdarmfisteln meistens mit Tuberkulose nichts zu tun haben. FreY und THoss können bezüglich des mikroskopischen Befundes nur in 5,3\% und bezüglich der allgemeinen Verhältnisse der Patienten nur in I I,9\% die Tuberkulose als Ursache der Mastdarmfistel anerkennen. R. VOLKMANN und F. R. KöNig dagegen sind der Ansicht, daB die Analfisteln in der Mehrzahl auf Tuberkulose zurückzuführen sind. De Quervain fand, daß die Hälfte aller Mastdarmfisteln tuberkulös seien und MeLchior kommt auf Grund langjähriger Beobachtungen zu dem Resultat, daß jede Mastdarmfistel, bei der nicht eine spezielle Ätiologie, Entwicklungsfehler, Trauma, Fremdkörper usw. - nachweislich in Betracht kommt, in dubio als tüberkulös aufzufassen ist.

Wir stimmen mit den meisten Autoren darin überein, daß bei weitem der größte Teil der Analfisteln tuberkulöser Natur ist. Zuverlässige statistische Angaben lassen sich darüber nicht machen, da man durch die bakteriologische und histologische Untersuchung, sowie durch den Tierversuch den spezifischen Charakter der Fistel nicht immer beweisen kann. In vielen Fällen wird man nur durch eine langjährige klinische Beobachtung den sicheren Zusammenhang der Analfistel mit der Tuberkulose bringen können.

Bei den Patienten unserer Anstalt war in Anbetracht ihres tuberkulösen Lungenleidens bei jeder Mastdarmfistel der Zusammenhang mit der Tuberkulose naheliegend, wenngleich man auch bei tuberkulösen Patienten nicht tuberkulöse Analfisteln beobachten kann. HARTMANN fand unter 626 Phthisikern $39 \mathrm{mal}=4,9 \%$ und zwar unter 447 Männern $6 \%$ unter I79 Frauen 3,35\% Analfisteln. Von den 489 Patienten, die im Jahre I92 I unsere Anstalt verließen, hatten I2 $=2,6 \%$ eine Analfistel. Daß diese Zahl hinter der von HARTMANN zurückbleibt, ist wohl in der Hauptsache dadurch bedingt, da $B$ bei den Patienten einer Privatheilanstalt ein größeres Reinlichkeitsbedürfnis und eine größere Aufmerksamkeit auf die Verdauungsfähigkeit vorliegt, wodurch ohne Zweifel die Gefahr der Analfistel herabgesetzt wird.

Die Frage der Ätiologie der Analfistel kann vor allem für die Therapie und Prognose derselben von großer Bedeutung sein. Die Behandlung der Fistel ist bisher fast ausschließlich operativ: Spaltung des Fistelganges in seiner ganzen Aus- dehnung; bei tuberliulösen. Fisteln eventuell energische Auslöffelung, Kauterisation oder ausgiebige Excision. Bei einer großen Anzahl von Mastdarmfisteln führt diese Behandlungsmethode zum Zicle. Für đie tuberkulösen Fisteln dagegen ist es oft geradezu charaliteristisch, daß sie nach der Operation gar nicht heilen oder sehr bald wieder käsig erweichen und aufbrechen. Von den I2 mit Analfistel behafteten Patienten des letzten Jahres waren drei zweimal und einer sogar viermal ohne Erfolg operiert worden. Eine durch operativen Eingriff geheilte Analfistel gehört bei unsern lungenkranken Patienten zu den Ausnahmen.

Diese Tatsache veranlaßte uns, zu versuchen, mit Röntgenstrahlen die Analfisteln zur Ausheilung zu bringen. Wenngleich sich unsere bisherigen Erfahrungen auf insgesamt $20 \mathrm{mit}$ Röntgenstrahlen behandelte Fälle beschränken, so darf man doch schon sagen, daß die Erfolge durchaus befriedigend waren und $z \mathbf{u}$ weiteren Versuchen berechtigen. Von den 12 Fällen des letzten Jahres kamen $6=50 \%$ zur völligen Ausheilung, darunter 3 Fälle, die 2 bzw. 4 mal ohne Erfolg operiert waren; bei den übrigen 6 bildete sich die Fistel wesentlich zurück, so daß sie kaum noch Erscheinungen machte und vielleicht durch spätere Bestrahlungen doch noch zur völligen Austrocknung kommen kann, zumal 3 dieser Patienten die Kur vorzeitig abbrechen mußten.

Technisch gingen wir so vor, daß wir wöchentlich 3 Bestrahlungen gaben und zwar für das Feld IO-I $5 \mathrm{x}=20-30 \%$ der H.E.D. bei $30 \mathrm{~cm}$. Fokusabstand und $4 \mathrm{~mm}$ Aluminiumfilter; runder Bleitubus von $5 \mathrm{~cm}$ Durchmesser; nach 3 Wochen Pause Wiederholung der Bestrahlungsserie; nach der 4. Bestrahlungsserie evtl. längere Pause.

Zusammenfassend dürfen wir feststellen: Die Mastdarmfistel ist in der Mehrzahl der Fälle tuberkulöser Natur. Die Erkennung der Ätiologie kann für die Therapie und Prognose von großer Bedeutung sein. Durch chirurgische Eingriffe läßt sich die tuberkulöse Mastdarmfistel in vielen Fällen nicht zum Verschluß bringen. Durch Röntgenbestrahlungen dagegen gelingt es sehr oft, die tuberkulöse Analfistel zur Austrocknung und Verheilung zu bringen.

Literatur: Melchion, Utber die Rolle der Tuberkulose als Ursache der Mastdarmfisteln. Berl. klin. Wochenschr. 19I7, Nr. 26. - Thoss, Über die Ursache der Mastdarmfisteln. Münch. med. Wochenschr. I920, Nr. 50.

\section{VASOMOTORISCH-TROPHISCHE STÖRUNGEN UND DEREN HEILUNG MITTELS PERIARTERIELLER SYMPATHEKTOMIE.}

Von

\author{
Heinrich Higier (Warschau).
}

Prof. F. BRÜNING bespricht in einer in der in Nr. I5, S. 729, der Klinischen Wochenschrift publizierten Abhandlung ${ }^{1}$ ) die Entstehungsweise trophischer Gewebsveränderungen. Auf die neuere Physiologie der spinalen Vasodilatatoren und der sympathischen Vasoconstrictoren hinweisend, gelangt er zum richtigen Schluß, daß die Nervenreizung gegenüber der Nervenlähmung in der Pathogenese der vasomotorischtrophischen Störungen die überragende Rolle spielt, und daß es daher bei konsequenter Behandlung dieser Störungen die Beseitigung oder wenigstens Minderung des krankhaften Reizzustandes zu erstreben sei. Er stützt sich hier auf günstige Resultate der neuen, von LÉrichE bei organischen Läsionen empfohlenen, von ihm nach Forsters Vorschlag bei einer vasomotorisch-trophischen Neurose nachgeprüften Methode der "periarteriellen Sympathektomie". Auf solche Weise gelingt es, manches Leiden der Heilung zuzuführen, das bisher als kaum beeinflußbar galt, bzw. zu verstümmelnden Eingriffen, wie Amputation, Veranlassung gab. „Wir sehen hier wiederum, schließt BRÜNING, wie aus einer anderen Auffassung über die Pathogenese eines Leidens uns ein neuer vielversprechender Weg für die Therapie gezeigt wird."

i) F. BRUNING. Nervenlähmung und Nervenreizung in ihrer Bedeutung für die Entstehung trophischer Gewebsveränderungen. 